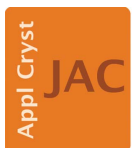

JOURNAL OF

APPLIED

CRYSTALLOGRAPHY

ISSN 1600-5767

Received 10 August 2021

Accepted 11 November 2021

Edited by T. J. Sato, Tohoku University, Japan

Keywords: neutron diffraction; hydrogenation; neutron instrumentation; solid-gas; metal hydrides; sapphire; powder diffraction.

Supporting information: this article has supporting information at journals.iucr.org/j

\section{A double-walled sapphire single-crystal gas- pressure cell (type III) for in situ neutron diffraction}

\author{
Raphael Finger, ${ }^{a}$ Thomas C. Hansen ${ }^{\mathrm{b}}$ and Holger Kohlmann ${ }^{\mathrm{a} *}$ \\ a'Inorganic Chemistry, Leipzig University, Johanisallee 29, Leipzig, 04103, Germany, and ${ }^{\mathbf{b}}$ Institut Laue-Langevin, \\ 71 avenue des Martyrs, Grenoble, 38000, France. *Correspondence e-mail: holger.kohlmann@uni-leipzig.de
}

In situ neutron diffraction is an important characterization technique for the investigation of many functional materials, e.g. for hydrogen uptake and release in hydrogen storage materials. A new sapphire single-crystal gas-pressure cell for elastic neutron scattering has been developed and evaluated; it allows conditions of $298 \mathrm{~K}$ and $9.5 \mathrm{MPa}$ hydrogen pressure and $1110 \mathrm{~K}$ at ambient pressure. The pressure vessel consists of a sapphire single-crystal tube of $35 \mathrm{~mm}$ radius and a sapphire single-crystal crucible as sample holder. Heating is realized by two $100 \mathrm{~W}$ diode lasers. It is optimized for the D20 diffractometer, ILL, Grenoble, France, and requires the use of a radial oscillating collimator. Its advantages over earlier sapphire single-crystal gas-pressure cells are higher maximum temperatures and lower background at low and high diffraction angles. The deuterium uptake in palladium was followed in situ for validation, proving the potential of the type-III gas-pressure cell for in situ neutron diffraction on solid-gas reactions.

\section{Introduction}

In situ X-ray and neutron diffraction have become very popular recently, because many interesting phenomena may be studied, such as phase transitions, chemical reactions, the wear of functional materials, the functioning of heterogeneous catalysts and battery materials, and industrial processes (Isnard, 2007; Pienack \& Bensch, 2011; Hansen \& Kohlmann, 2014; Yang et al., 2016; Peterson et al., 2017; Kohlmann, 2019). Time-resolved in situ neutron diffraction investigations are particularly interesting for materials in energy storage because processes of charging and discharging may be studied on an atomic level, e.g. in battery materials or in metal hydrides for hydrogen storage. Many phase transitions occur within the latter class of compounds, e.g. temperature- and pressuredriven structural and magnetic phase transitions (Kohlmann et al., 2003; Goncharenko et al., 1996). Hydrogen uptake and release from metal hydrides are of importance in view of the use as hydrogen stores (Møller et al., 2014; Hansen \& Kohlmann, 2014; Götze et al., 2018).

For the in situ investigation of hydrogenation reactions under hydrogen (deuterium) gas pressure and at elevated temperature, specialized gas-pressure cells with laser heating have been developed and used in many studies (Peterson et al., 2017; Götze et al., 2018; Finger, Kurtzemann et al., 2021; Finger, Hansen \& Kohlmann, 2021). A sample environment based on single-crystal holders made of $\alpha-\mathrm{Al}_{2} \mathrm{O}_{3}$ [called sapphire herein; see Finger, Kurtzemann et al. (2021)] allows for extremely low background without parasitic reflections, given proper orientation of the single crystal. Typically, they operate with laser heating at moderate temperatures and 
allow for a time resolution of less than $1 \mathrm{~min}$ (on high-intensity diffractometers). They can withstand high hydrogen (deuterium) gas pressures [e.g. 16.0 MPa at $298 \mathrm{~K}$ and $8.0 \mathrm{MPa}$ at $655 \mathrm{~K}$ for type I (Finger, Kurtzemann et al., 2021)], but are not designed for high temperatures. The highest temperature reached so far, $718 \mathrm{~K}$, was recorded at ambient pressure with a type-II cell (Finger, Hansen \& Kohlmann, 2021). In the present publication we describe a new type of sapphire singlecrystal gas-pressure cell (type III). It allows for in situ neutron powder diffraction at considerably higher temperatures, thus enabling a wider range of solid-gas reactions to be studied. It is optimized for the D20 diffractometer (Hansen et al., 2008), ILL, Grenoble, France, in high-resolution mode, with a takeoff angle of $120^{\circ}$ and a wavelength of about $187 \mathrm{pm}$, and requires the use of a radial oscillating collimator.

\section{Concept, pressure vessel and sample holder of the type-III gas-pressure cell}

The previously presented gas-pressure cells of types I and II combine pressure vessel and sample holder in one work piece, a $10 \mathrm{~cm}$-long machined sapphire single crystal with a $6 \mathrm{~mm}$ borehole (Finger, Kurtzemann et al., 2021; Finger, Hansen \& Kohlmann, 2021). In type-I gas-pressure cells the sample holder is fixed between a base mount and a flange joint with no connection in between, thus providing free optical access in the diffraction plane (Finger, Kurtzemann et al., 2021). The type-II gas-pressure cell uses a single corpus around the sample holder with windows for primary and scattered neutron beams, optical surveillance and laser heating (Finger, Hansen \& Kohlmann, 2021). For the cell presented herein, type III, the tasks of holding pressure and holding a sample are uncoupled, i.e. the pressure vessel and sample holder are two different work pieces. This results in more degrees of freedom for physical dimensions and materials, and offers the opportunity to optimize both pieces for their individual task. For example, the sample holder may have a smaller wall thickness, since it does not need to withstand the gas pressure, reducing the contribution of the sample holder and thus resulting in a higher quality of the diffraction data. Furthermore, a smaller wall thickness for the sample holder means that a much wider variety of materials can be used as sample holder, e.g. parasitic reflections from polycrystalline materials or the structured background of amorphous materials may be small or negligible. Such materials were ruled out of consideration as a pressure vessel by Finger et al. but meet the requirements to be potential sample holders for the type-III gas-pressure cell. Therefore, we firstly focused on quartz glass (Hilgenberg $\mathrm{GmbH}$, Malsfeld, Germany) as it is available in small wall thicknesses of less than $1 \mathrm{~mm}$, even with an inner diameter of $4 \mathrm{~mm}$. Though quartz glass showed a highly structured background despite its small wall thickness of $0.25 \mathrm{~mm}$ (Fig. 1, top), a sapphire single-crystal crucible, $32 \mathrm{~mm}$ in height, $6 \mathrm{~mm}$ inner diameter with a wall thickness of $1 \mathrm{~mm}$, has been chosen as sample holder (Fig. 2). Despite the much higher wall thickness, the crucible offers a better overall data quality because of its low background contribution for proper orientation of the single-crystal crucible. For the pressure vessel, a sapphire single-crystal tube is used (Impex HighTech $\mathrm{GmbH}$, Münster, Germany). The material is a common choice for in situ diffraction experiments (Finger, Kurtzemann et al., 2021; Götze et al., 2018). Neutron activation, neutron absorption for thermal neutrons and incoherent scattering are low for sapphire (Sears, 1992). Furthermore, potential irradiation damage occurs only at neutron doses as high as $10^{18}$ neutrons $\mathrm{cm}^{-2}$ (Abdukadyrova, 2005), which is way above the expected value for a typical neutron diffraction experiment considering the neutron flux at D20 $\left[10^{7}\right.$ neutrons s $\mathrm{cm}^{-1} \mathrm{~cm}^{-2}$ for $\lambda=1.87 \AA$, Ge(115) monochromator, $120^{\circ}$ take-off angle (Hansen et al., 2008)].

To completely avoid the contribution of the pressure vessel to the resulting diffraction pattern, a minimum diameter of $35 \mathrm{~mm}$ is dictated by the radial oscillating collimator used at D20 (Fig. 2). Equation (1) of Finger, Kurtzemann et al. (2021) gives a maximum pressure of $63 \mathrm{MPa}$ at $298 \mathrm{~K}$ for a wall thickness of $3 \mathrm{~mm}$ and about $43 \mathrm{MPa}$ at $770 \mathrm{~K}$. Including a safety factor of four to account for mechanical stress and potential imperfections of the single crystal, the use of the type-III gas-pressure cell is limited to a maximum overpressure of $10 \mathrm{MPa}$. So far, a maximum pressure of $9.5 \mathrm{MPa}$ at room temperature has been tested. Higher pressures may be realized but have not yet been tested, because they are not necessary for the kind of in situ experiment that we have so far
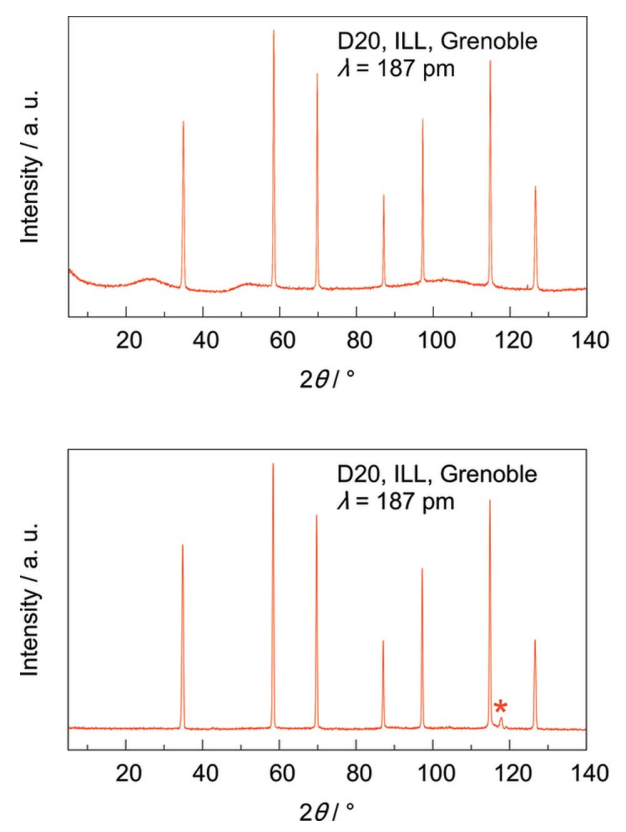

Figure 1

Neutron powder diffraction data of silicon powder in a quartz glass tube with an inner diameter of $4 \mathrm{~mm}$ and a wall thickness of $0.25 \mathrm{~mm}(5 \leq 2 \theta \leq$ $140^{\circ}$, normalized to have the same maximum intensity as the strongest reflection, 5 min measurement time; NUMOR 975353; https://doi.org/ 10.5291/ILL-DATA.5-24-605) (top) and in a type-III gas-pressure cell with a sapphire single-crystal crucible as sample holder with an inner diameter of $6 \mathrm{~mm}$ and a wall thickness of $1 \mathrm{~mm}$ (parasitic reflection from the sample environment marked with $*, 5 \leq 2 \theta \leq 140^{\circ}, 10 \mathrm{~min}$ measurement time; NUMOR 178819; https://doi.org/10.5291/ILL-DATA. 5-24-639) (bottom). (NUMOR is the internal raw data labelling of the ILL, Grenoble, France.) 
carried out, typically with $0.1<p<$ $10 \mathrm{MPa}$ and $300<T<700 \mathrm{~K}$.

\section{Double-walled sapphire single- crystal gas-pressure cell (type III)}

The gas-pressure cell has three main parts: a bottom plate, a corpus and a top plate. All main parts are made of austenitic chromium-nickel stainless steel (EN 1.4301/AISI 304). The bottom plate has a diameter of $80 \mathrm{~mm}$ and a thickness of $8 \mathrm{~mm}$. The corpus is connected to it via eight M6 Allen screws. To fix the cell on the diffractometer, the bottom plate has an ISO metric M8 thread (Fig. 2). The grooves in the plate, 36.17 and $41.41 \mathrm{~mm}$, are for the NBR 70 O-ring seal (Nitrile Butadiene Rubber 70 Shore, usable up to $393 \mathrm{~K}$ ) to realize gas pressure. The outer rim keeps the O-ring in position. The corpus is the main part of the gas-pressure cell as it connects the bottom and top plates. It is painted with neutron-absorbing gadolinium oxide varnish to prevent neutron activation. The top plate is similar to the bottom plate with six boreholes to screw it to the corpus. A T-piece is welded on top of the plate for gas feed and temperature measurement. A thermocouple covered with neutron-absorbing gadolinium oxide varnish may be screwed into a $6 \mathrm{~mm}$ Swagelok nut in order to measure temperature in the reaction chamber (Figs. 3 and 4). Temperature surveillance with a pyrometer as for types I and II did not work, because only the temperature of the pressure vessel was measured. However, the opening that was originally designed for the pyrometer can be used for optical surveillance, which is valuable for reactions including colour changes.

The higher gas volume, compared with the type-I and -II cells, is compensated with an inlet, lowering the gas volume from 67.3 to $27.3 \mathrm{~cm}^{3}$ and thus minimizing risk. The inlet is $32 \mathrm{~mm}$ in diameter and $60 \mathrm{~mm}$ in height and has a $16 \mathrm{~mm}$ opening on the bottom for the sample holder and its rest. So as not to interfere with the primary beam or scattered neutrons, the inlet has the same openings as the corpus (Figs. 3 and 4). To prevent neutron activation, the corpus and inlet are painted with a neutron-absorbing varnish as used for the typeII gas-pressure cells (Finger, Hansen \& Kohlmann, 2021). The inlet is fixed on the bottom plate via three pins, allowing a reproducible positioning. The central rest for the sample holder is not higher than the painted inlet, thus preventing neutron activation.

\section{Assembly}

As a first step, an O-ring seal (Fig. 5, black) is placed onto the bottom plate (Fig. 5, red). Then, the corpus (Fig. 5, green) is screwed to the bottom plate. The crucible is placed on the sample stage in the middle of the bottom plate and the inlet (Fig. 5, blue) is carefully put on top, being clicked into the

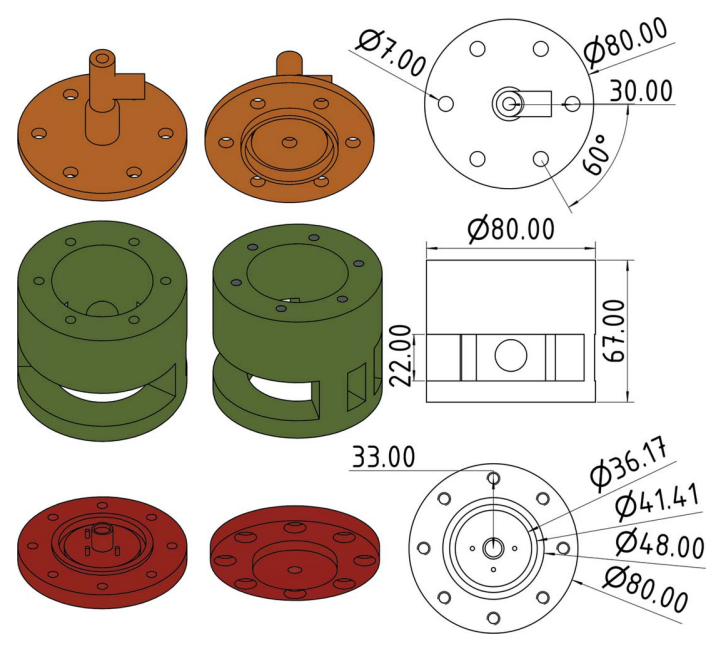

Figure 3

Main parts of the type-III gas-pressure cell: top plate (top), corpus (middle), bottom plate (bottom); length specification in $\mathrm{mm}$.
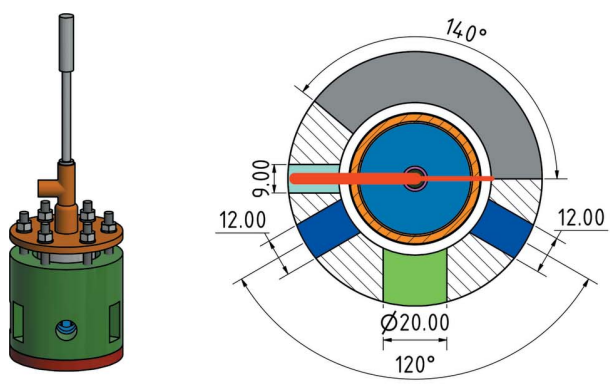

Primary beam

Window for primary beam

Sample

Window for scattered

neutrons

Windows for laser heating

Window for pyrometer of optical surveillance

Sample holder

Pressure vessel

Inlet
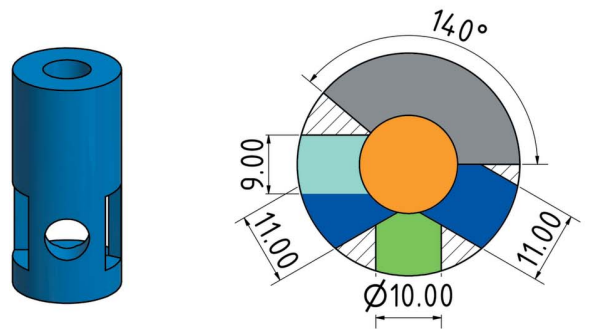

Window for primary beam Window for scattered

Windows for laser heating Window for pyrometer or optical surveillance

Opening for sample holde

Figure 4

Type-III gas-pressure cell (top) and aluminium inlet (bottom) in threedimensional representation (left), and section of the plane of diffraction (right); length specification in $\mathrm{mm}$. 


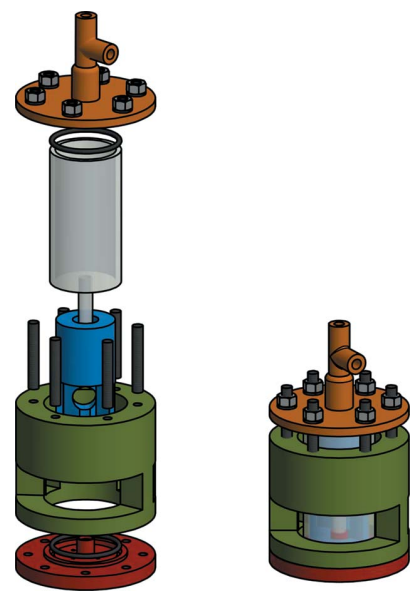

Figure 5

Exploded-view drawing (left) and three-dimensional representation (right) of the type-III gas-pressure cell.

three pins (Fig. 3, bottom left) to allow a reproducible positioning. Afterwards, the sapphire tube as pressure vessel (Fig. 5, grey) is placed on the O-ring. The threaded bars (ISO metric M6, $40 \mathrm{~mm}$ long) are turned all the way in the corresponding threaded boreholes on the top of the corpus. The upper seal is positioned on the top of the sapphire tube and the top plate is put carefully on top. Finally, the gas-pressure cell is screwed together by fastening the nuts on the threaded bars finger tight and then turning them crosswise step by step up to a torque of $1.2 \mathrm{~N} \mathrm{~m}$. The thermocouple and gas supply may be attached afterwards. To realize the gas-tight sealing of the thermocouple with an inner diameter of $6 \mathrm{~mm}$, a corresponding $6 \mathrm{~mm}$ Swagelok screw connection is attached and fixed.

\section{Tested temperature and pressure conditions}

In the course of our in situ studies, typical temperaturepressure conditions for hydrogenation reactions were applied, which are considerably lower than the maximum values given above. As breaking tests were not performed, we cannot state the maximum temperature-pressure conditions, but only those tested and found to be safe (Table 1 and Appendix $A$ ). The maximum temperature-pressure conditions might be
Table 1

Maximum gas-pressure $(p)$ and temperature $(T)$ conditions tested for the type-III sapphire single-crystal gas-pressure cell with a sapphire tube of $35 \mathrm{~mm}$ inner diameter and $3 \mathrm{~mm}$ wall thickness as pressure vessel.

\begin{tabular}{llr}
\hline Gas & $p(\mathrm{MPa})$ & $T(\mathrm{~K})$ \\
\hline Air & 0.1 & 1110 \\
$\mathrm{D}_{2}$ & 2.0 & 360 \\
$\mathrm{H}_{2}$ & 9.5 & 298 \\
\hline
\end{tabular}

higher. The temperatures are much higher than for type-I and -II cells (Finger, Kurtzemann et al., 2021; Finger, Hansen \& Kohlmann, 2021), which is clearly one of the advantages of decoupling the pressure vessel and sample holder, albeit at the cost of reduced maximum pressure. For technical reasons, high temperatures have so far only been tested in air, but are expected to hold also for hydrogen pressures.

For the exact sample temperature determination on a neutron diffractometer, calibration was performed via the known thermal expansion of the palladium lattice (Dutta \& Dayal, 1963) by a procedure described previously (Finger, Kurtzemann et al., 2021).

\section{Validation of the double-walled sapphire single- crystal gas-pressure cell}

An $\omega$ scan (data collection while turning around the principal goniometer axis) of the type-III gas-pressure cell (Fig. 6, left) shows a section of about $5^{\circ}$ with no significant change in absolute intensities (Fig. 6, middle) and of about $8^{\circ}$ with unaffected reflection/background ratio (Fig. 6, right). Typically, less than $5^{\circ}$ is needed for the fine tuning of the $\omega$ position, according to our experience with the single crystals used in type-I and -II cells (Finger, Kurtzemann et al., 2021; Finger, Hansen \& Kohlmann, 2021). Thus, the window is wide enough to allow for proper alignment of the type-III gas-pressure cells.

To validate the type-III gas-pressure cell, the hydrogenation of palladium was studied. It is well suited due to a great body of knowledge, the high symmetry of the starting material and the resulting palladium deuteride, and favourable neutron scattering lengths. For the reaction, deuterium was chosen instead of hydrogen because of its lower incoherent scattering cross section. Palladium powder $(99.95 \%$, mesh size $<150 \mu \mathrm{m}$,
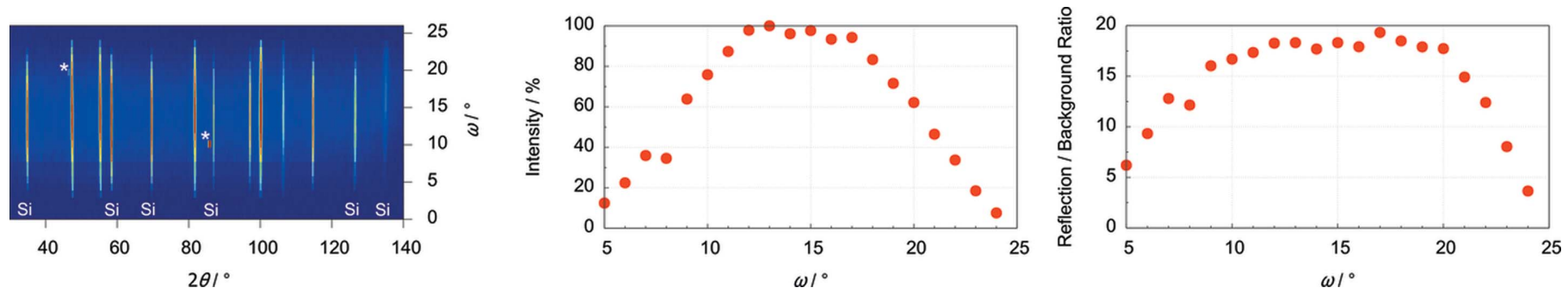

Figure 6

False-colour plot of an $\omega$ scan of silicon powder in an aluminium crucible performed on D20 at ILL in $1^{\circ}$ steps of $\omega$ and with $60 \mathrm{~s} \mathrm{data}$ accumulation per $\omega$ position. Highest intensity in red, lowest intensity in blue, parasitic reflection from the sample environment marked with * (left). Relative intensities of the silicon 111 reflection as a function of $\omega$ (middle) and reflection/background ratio as a function of $\omega$ (right). (NUMOR 138794; http://doi.org/10.5291/ ILL-DATA.5-24-621). 


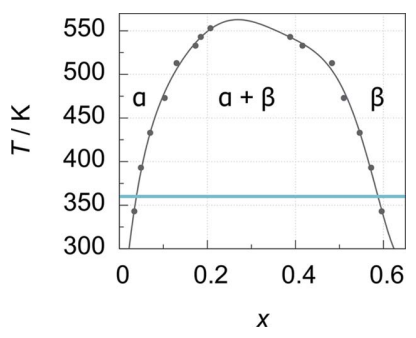

Figure 7

Palladium-deuterium phase diagram (temperature versus deuterium content $x$ in $\mathrm{PdD}_{x}$ ); based on absorption isotherms [data from Wicke \& Blaurock (1987); grey points] fitted with a polynomial function (grey line) and the temperature used for validation of the gas-pressure cell (blue line).

GoodFellow) was dried under vacuum (1 Pa) at $773 \mathrm{~K}$ for $24 \mathrm{~h}$ and handled under argon afterwards. In the in situ experiment, palladium powder was heated under vacuum $(9 \mathrm{kPa})$ to 360 (2) K before injections of deuterium gas were slowly added. Rietveld refinements were performed using FullProf with pseudo-Voigt profile functions (Rodríguez-Carvajal, 1993). Starting models for palladium and the $\alpha$ and $\beta$ phases of palladium deuteride were taken from the work of Kohlmann et al. (2013).

When hydrogen is absorbed by palladium, hydrogen atoms occupy octahedral voids; tetrahedral void occupation as seen above the critical point of the phase diagram (Pitt \& Gray, 2003) was not observed, as we performed the experiment at
Table 2

Crystal structures of palladium deuterides $\mathrm{PdD}_{x}$ at 360 (2) $\mathrm{K}$ and between 0.085 and $2.0 \mathrm{MPa} \mathrm{D}_{2}$ pressure (space-group type $F m \overline{3} m$, Pd in $4 a 000$, D in $\left.4 c \frac{1}{2} \frac{1}{2} \frac{1}{2}\right)$.

\begin{tabular}{llllll}
\hline Phase & $\begin{array}{l}p\left(\mathrm{D}_{2}\right) \\
(\mathrm{MPa})\end{array}$ & $\begin{array}{l}\text { Lattice } \\
\text { parameter }(\AA)\end{array}$ & $\begin{array}{l}\text { Deuterium } \\
\text { content } x\end{array}$ & $\begin{array}{l}B_{\text {iso }}(\mathrm{Pd}) \\
\left(\AA^{2}\right)\end{array}$ & $\begin{array}{l}B_{\text {iso }}(\mathrm{D}) \\
\left(\AA^{2}\right)\end{array}$ \\
\hline$\alpha-\mathrm{PdD}_{x}$ & 0.085 & $3.90411(3)$ & $0.024(2)$ & $1.18(4)$ & $4 \dagger$ \\
$\alpha-\mathrm{PdD}_{x}$ & 0.25 & $3.90681(4)$ & $0.033(4)$ & $1.41(5)$ & $4 \dagger$ \\
$\beta-\mathrm{PdD}_{x}$ & 0.25 & $4.03749(15)$ & $0.560(5)$ & $1.39(7)$ & $3.85(14)$ \\
$\beta-\mathrm{PdD}_{x}$ & 2.0 & $4.04788(9)$ & $0.626(6)$ & $1.35(6)$ & $4.09(9)$ \\
\hline
\end{tabular}

$\dagger$ Fixed as no proper refinement was possible due to the small amount of deuterium, value taken from type-II validation (Finger, Hansen \& Kohlmann, 2021).

temperatures and pressures below the critical point. The deuterium contents of palladium deuteride in this in situ experiment (Table 2) are in good accordance with the experimental data (Wicke \& Blaurock, 1987) (Figs. 7, 8 and 9). The lattice parameter and isotropic displacement parameters $\left(B_{\text {iso }}\right)$ of the atoms are reasonable (Table 2).

\section{Data quality of the type-I, -II and -III gas-pressure cells}

To evaluate the overall data quality, the background at low and high $2 \theta$ values and the reflection/background ratios are considered (Table 3 and Fig. 10, top). The same amount of
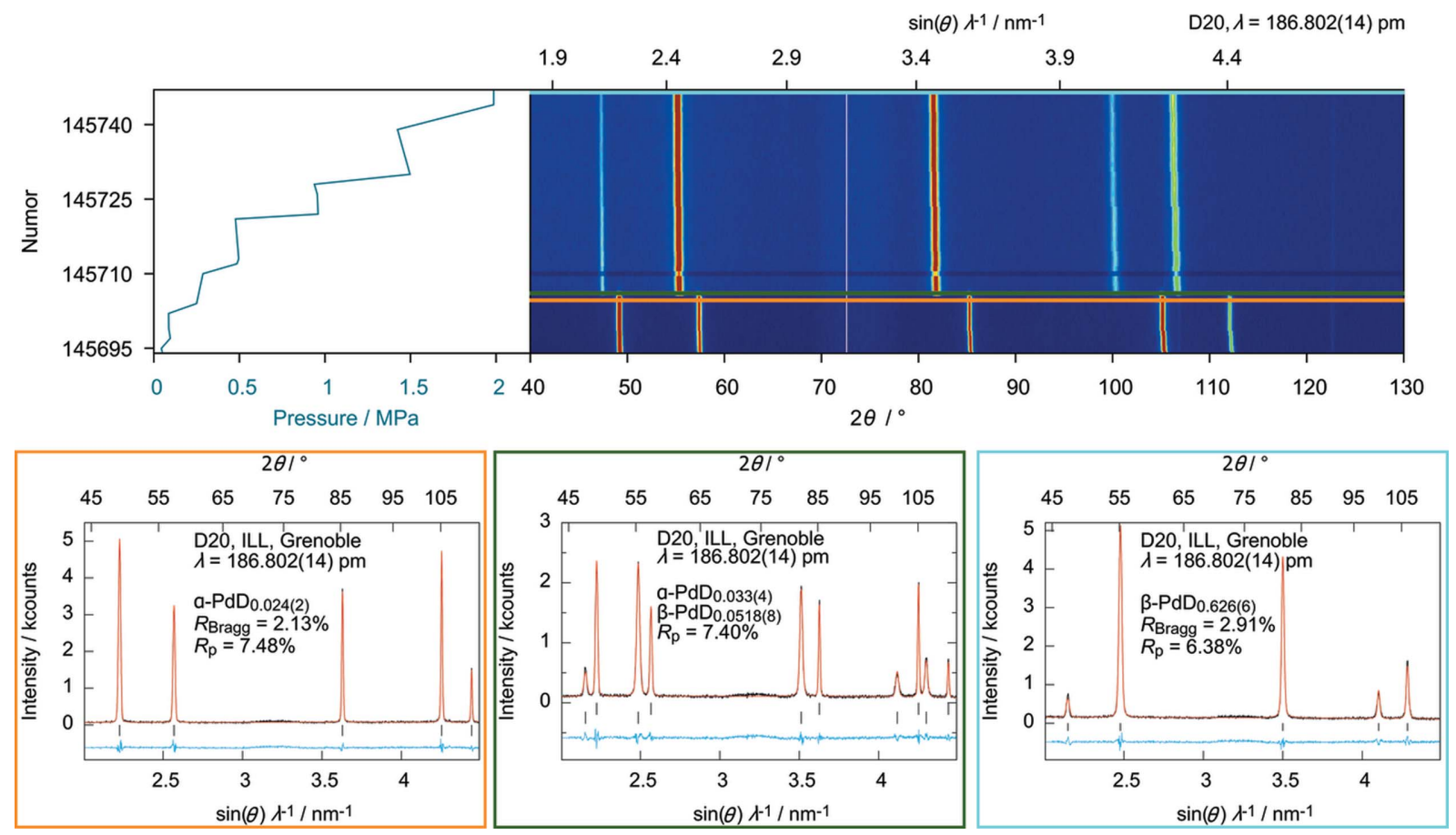

Figure 8

In situ neutron powder diffraction of the reaction of palladium powder with deuterium at 360 (2) K. Top: pressure profile (left), diffraction data as falsecolour plot with highest intensity in red and lowest intensity in blue (right). Bottom: Rietveld refinements (observed intensities, $I_{\mathrm{obs}}$, in black, calculated intensities, $I_{\text {calc }}$, in red, difference, $I_{\text {obs }}-I_{\text {calc }}$, in blue, excluded region $72.6 \leq 2 \theta \leq 72.7^{\circ}$ due to detector failure, 2 min per NUMOR; https://doi.org/ 10.5291/ILL-DATA.5-22-767) of the crystal structures of $\alpha-\mathrm{PdD}_{0.024}$ (2) (NUMOR 145703 , left, corresponding to orange line top right), $\alpha$-PdD 0.033 (4) and $\beta$-Pd $\mathrm{Pd}_{0.518(8)}$ (NUMOR 145704, middle, corresponding to dark-green line top right), and $\beta$-PdD $\mathrm{D}_{0.626(6)}$ (NUMOR 145748, right, corresponding to lightblue line top right). 
Table 3

Reflection/background ratio $R_{\mathrm{I}}$ of all silicon reflections measured from neutron powder diffraction data using the type-III gas-pressure cell.

\begin{tabular}{lrl}
\hline$h k l$ & $2 \theta\left(^{\circ}\right)$ & $R_{\mathrm{I}}$ \\
\hline 111 & 34.85 & $25.8(3)$ \\
220 & 58.35 & $33.0(4)$ \\
311 & 69.75 & $29.7(4)$ \\
400 & 87.10 & $11.3(2)$ \\
331 & 97.25 & $19.4(3)$ \\
422 & 114.90 & $27.1(3)$ \\
$511 / 333$ & 126.65 & $13.6(3)$ \\
\hline
\end{tabular}

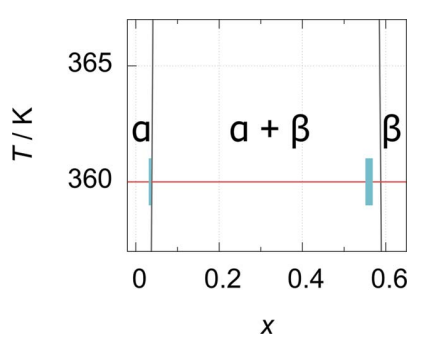

Figure 9

Part of the palladium-deuterium phase diagram (temperature versus deuterium content $x$ in $\mathrm{PdD}_{x}$ ): blown-up section of Fig. 7 at $0.25 \mathrm{MPa}$ deuterium gas pressure, data from Wicke \& Blaurock (1987), experimentally determined deuterium content at $0.25 \mathrm{MPa}$ and 360 (2) K (red line) for $\alpha$ - $\mathrm{PdD}_{0.033(4)}$ and $\beta$ - $\mathrm{PdD}_{0.560(5)}$ with \pm as blue boxes.
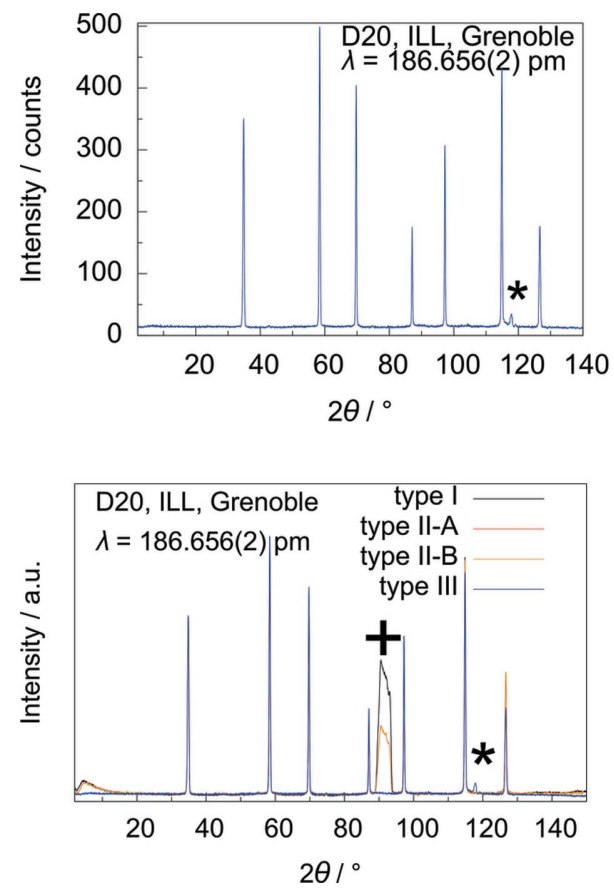

Figure 10

Neutron diffraction data of the type-III gas-pressure cell with silicon powder $\left(2 \leq 2 \theta \leq 140^{\circ}\right.$, parasitic reflection marked with *, 10 min measurement time; NUMOR 178819) (top), and comparison of the normalized neutron diffraction data for type-I, -II and -III gas-pressure cells (sample holder contribution from type-I and -II sample holders marked with + , parasitic reflection of the type-III sample environment marked with *; NUMORs 178776, 178781, 178810, 178819) (bottom) (https://doi.org/10.5291/ILL-DATA.5-24-639).

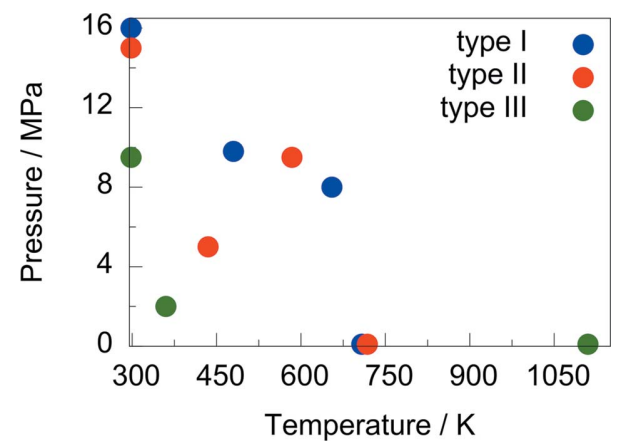

Figure 11

Comparison of tested temperature and pressure conditions with type-I (Finger, Kurtzemann et al., 2021), type-II (Finger, Hansen \& Kohlmann, 2021) and type-III (this work) gas-pressure cells.

sample has been used for measurements with the type-I and -II gas-pressure cells, and only the measurement time is different (10 min for type III instead of $20 \mathrm{~min}$ for types I and II); thus the measurements are comparable for normalized representations (Fig. 10, bottom). Details for the type-I and -II measurements are provided by Finger, Hansen \& Kohlmann (2021). The striking feature of the type-III compared with the type-I and -II gas-pressure cells is a very low background at low and high angles [calculated as by Finger, Hansen \& Kohlmann (2021), standard deviation of intensities considered]. The air and deuterium gas scattering, visible at low $2 \theta$ angles, is very small, as a result of the inlet (Figs. 4 and 5).

The tested temperature and pressure conditions of all three types of gas-pressure cells are compared in Fig. 11. The type-I cell has been used for over ten years and thus has well known operating conditions. Type-II and -III cells are mainly used for laboratory tests and are less well studied under real conditions. The type-III cell offers most potential at higher temperatures with a maximum of about $1110 \mathrm{~K}$ reached at $0.1 \mathrm{MPa}$ air.

\section{Conclusions}

In this work we present a new design for a sapphire singlecrystal gas-pressure cell for in situ neutron diffraction. It is suitable for hydrogenation reactions up to $10 \mathrm{MPa}$ and reached a maximum temperature of about $1110 \mathrm{~K}$. Its advantage over earlier designs is a lower diffraction background at low and high diffraction angles. The decoupling of the sample holder and pressure vessel allows for an optimization of both parts independently. Moreover, the new design allows for higher sample temperatures and thus enables further studies of thermal stability, decomposition, and reversibility of hydrogen uptake and release. The adaptation to gases other than hydrogen should be straightforward and will allow a wide range of in situ experiments on solid-gas reactions in the future.

\section{APPENDIX $A$}

\section{Validation of temperature measurements}

In order to confirm the measured temperatures in the type-III gas-pressure cell, three different experiments were performed 


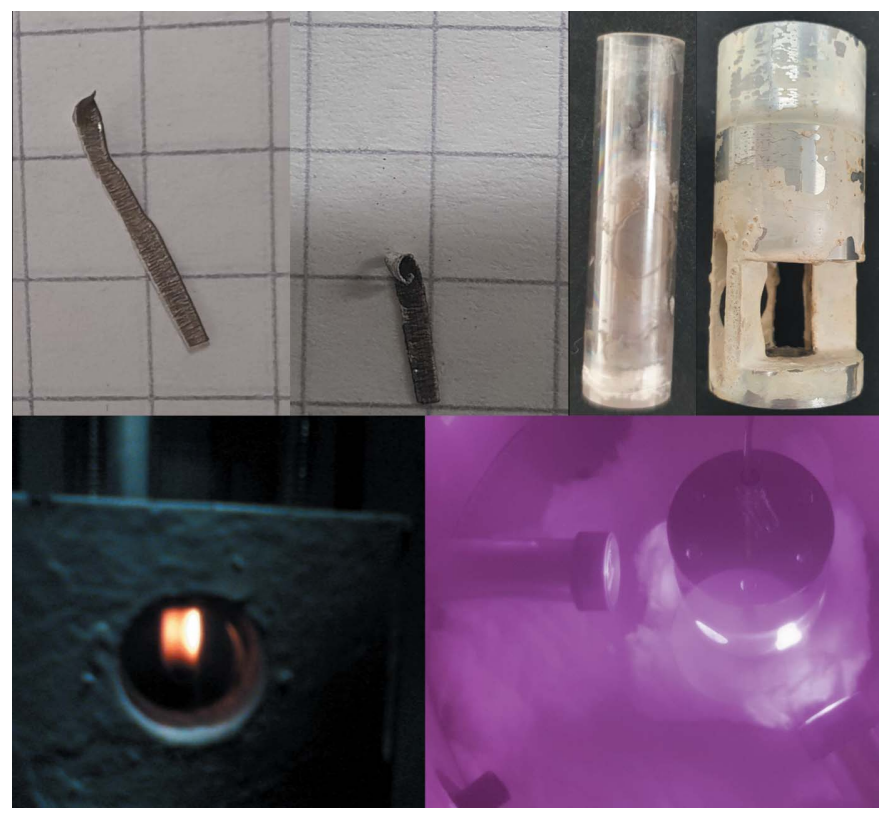

Figure 12

Photographs of the type-III gas-pressure cell and in situ setup during temperature tests: the piece of aluminium before and after experiment $\mathrm{C}$ (see Appendix $A$ ) (top left), crucible with the aluminium melted into the silicon and inlet after experiment $\mathrm{C}$ (top right), webcam view through an infrared absorbing glass filter along the pyrometer window at $1110 \mathrm{~K}$ and a laser power of $91.5 \mathrm{~W}$ during experiment $\mathrm{A}$ (bottom left), and the in situ setup at $803 \mathrm{~K}$ and a laser power of $62.6 \mathrm{~W}$ without filters taken with a mobile phone (bottom right).

using the same crucible with a 20-22 mm-high filling of silicon powder and heating by two diode lasers running at $91.5 \mathrm{~W}$. A thin thermocouple was put into the crucible, measuring the temperature at the position of maximum laser heating to be $1110 \mathrm{~K}$ (experiment A). The temperature of the top surface of the sample was measured by a pyrometer to be $1013 \mathrm{~K}$ (experiment B). A long thin piece of aluminium was stuck into the silicon powder, reaching from the position of maximum laser heating to the air above the heating zone, while a thermocouple at $3 \mathrm{~mm}$ depth measured $839 \mathrm{~K}$ (experiment $\mathrm{C}$ ). About half of the aluminium melted, which is in accordance with the temperature gradient and proves that the maximum temperature was at least as high as the melting point of aluminium $(933 \mathrm{~K})$. Additionally, the gadolinium oxide varnish showed signs of thermal decomposition, which is another indication of high sample temperatures (Fig. 12).

\section{Acknowledgements}

We acknowledge the Institut Laue-Langevin for provision of beamtime at the high-intensity powder diffractometer D20 (Finger, Hansen \& Kohlmann, 2021; http://10.5291/ILLDATA.1-10-47). Open access funding enabled and organized by Projekt DEAL. Author contributions are as follows: conceptualization, RF, HK; methodology, RF, HK; software, RF; validation, RF; formal analysis, RF; investigation, RF, $\mathrm{TCH}, \mathrm{HK}$; resources, HK; data curation, RF; writing - original draft preparation, RF; writing - review and editing, RF, TCH, HK; visualization, RF; supervision, HK; project administration, HK; funding acquisition, HK.

\section{Funding information}

This work was supported by the BMBF - German Federal Ministry of Education and Research (grant No. 05K16OL1 to Holger Kohlmann).

\section{References}

Abdukadyrova, I. Kh. (2005). Inorg. Mater. 41, 1080-1084.

Dutta, B. N. \& Dayal, B. (1963). Phys. Status Solidi B, 3, 2253-2259.

Finger, R., Hansen, T. C. \& Kohlmann, H. (2021). QuBS, 5, 22.

Finger, R., Kurtzemann, N., Hansen, T. C. \& Kohlmann, H. (2021). J. Appl. Cryst. 54, 839-846.

Goncharenko, I. N., Mignot, J.-M. \& Mirebeau, I. (1996). Neutron News, 7(3), 29-31.

Götze, A., Auer, H., Finger, R., Hansen, T. C. \& Kohlmann, H. (2018). Physica B, 551, 395-400.

Hansen, T. C., Henry, P. F., Fischer, H. E., Torregrossa, J. \& Convert, P. (2008). Meas. Sci. Technol. 19, 034001.

Hansen, T. C. \& Kohlmann, H. (2014). Z. Anorg. Allg. Chem. 640, 3044-3063.

Isnard, O. (2007). C. R. Phys. 8, 789-805.

Kohlmann, H. (2019). Eur. J. Inorg. Chem. 2019, 4174-4180.

Kohlmann, H., Kurtzemann, N. \& Hansen, T. (2013). Powder Diffr. 28, S242-S255.

Kohlmann, H., Moyer, R. O. Jr, Hansen, T. \& Yvon, K. (2003). J. Solid State Chem. 174, 35-43.

Møller, K. T., Hansen, B. R. S., Dippel, A.-C., Jørgensen, J.-E. \& Jensen, T. R. (2014). Z. Anorg. Allg. Chem. 640, 3029-3043.

Peterson, V. K., Auckett, J. E. \& Pang, W.-K. (2017). IUCrJ, 4, 540-554.

Pienack, N. \& Bensch, W. (2011). Angew. Chem. 123, 2062-2083.

Pitt, M. P. \& Gray, E. (2003). Europhys. Lett. 64, 344-350.

Rodríguez-Carvajal, J. (1993). Physica B, 192, 55-69.

Sears, V. F. (1992). Neutron News, 3(3), 26-37.

Wicke, E. \& Blaurock, J. (1987). J. Less-Common Met. 130, 351-363. Yang, J., Muhammad, S., Jo, M. R., Kim, H., Song, K., Agyeman, D. A., Kim, Y.-I., Yoon, W.-S. \& Kang, Y.-M. (2016). Chem. Soc. Rev. 45, 5717-5770. 